\title{
B-catenin expression in relation to genetic instability and prognosis in colorectal cancer
}

\author{
ANN MÅRTENSSON ${ }^{1}$, ̊̊KE ÖBERG ${ }^{3}$, ANDREAS JUNG ${ }^{4}$, KRISTINA CEDERQUIST $^{2}$, \\ ROGER STENLING ${ }^{1}$ and RICHARD PALMQVIST ${ }^{1}$
}

\begin{abstract}
Departments of ${ }^{1}$ Medical Biosciences, Pathology, ${ }^{2}$ Clinical Genetics and ${ }^{3}$ Surgical and Perioperative Sciences, Surgery, Umeå University, Umeå, Sweden; ${ }^{4}$ Department of Pathology, Institut für Pathologie, LMU, D-80337 München, Germany
\end{abstract}

Received June 29, 2006; Accepted September 20, 2006

\begin{abstract}
In the carcinogenesis of colorectal cancer (CRC) genetic instability and dysfunction of the Wnt-signalling pathway play important roles. Most Wnt-signalling dysfunctions lead to the nuclear accumulation of $\beta$-catenin. The aim of the present study was to investigate whether nuclear accumulation of $\beta$-catenin is associated with prognosis and genetic instability. We used immunohistochemistry to study nuclear ß-catenin expression in 67 CRCs. The expression was evaluated in the entire tumour section as mean values and in tumour budding at the invasive margin. We compared the results with chromosomal and microsatellite instability (CIN vs. MSI), p53 accumulation, and clinicopathological variables including survival. The nuclear accumulation of $\beta$-catenin was significantly associated with abnormal p53 expression and aneuploidy, typically for CIN, whereas no tumour with nuclear B-catenin expression at the invasive margin displayed MSI. The B-catenin expression pattern did not correlate significantly with CRC patient prognosis when including all stages. However, in the clinically most interesting prognostic group, Dukes' stage B patients, high nuclear accumulation of $\beta$-catenin was associated with a poor prognosis $(\mathrm{p}=0.01)$. Our results suggest that nuclear accumulation of $\beta$-catenin in CRC is related to CIN and may be of prognostic importance. However, larger studies are needed to verify these findings.
\end{abstract}

\section{Introduction}

Colorectal cancer remains the second leading cause of cancer deaths in Western Countries. In contrast to many other malignancies the survival rate has not changed significantly during the last three decades (1).

Colorectal cancer (CRC) develops along two distinct pathways: chromosomal instability (CIN) or microsatellite instability (MSI) (2-4). Up to $85 \%$ of colorectal tumours show chromosomal instability (CIN) which is accompanied by

Correspondence to: Dr Richard Palmqvist, Department of Medical Biosciences, Pathology, Bld 6M, 2nd floor, Umeå University, SE-901 85 Umeå, Sweden

E-mail: richard.palmqvist@medbio.umu.se

Key words: $ß$-catenin, genetic instability, colorectal cancer, prognosis accumulation of somatic mutations in tumour suppressor genes as TP53 or the adenomatous polyposis coli (APC) and oncogenes such as $\beta$-catenin and $K R A S(3,5,6)$. On the other hand, MSI is caused by an impaired capacity of the DNA mismatch repair system and only $10-15 \%$ of sporadic colorectal cancer show MSI. These cancers are mostly diploid and show no gross chromosomal changes (7).

In sporadic CRC the overall frequency of APC mutation is around $60 \%$, leading to loss of degradation of its downstream target, $B$-catenin, the main effector of the Wnt pathway, causing nuclear translocation and constitutive overexpression (8-10).

Normally, APC forms a multimolecular complex with $ß$-catenin, Axin/Conductin and GSK3ß, to target cytoplasmic $\beta$-catenin for proteasomal degradation. Since most APC mutations result in truncated proteins lacking most of the binding domains for $\beta$-catenin and/or Axin, no efficient degradation can take place, and thus B-catenin will accumulate $(9,10)$. Although about $40 \%$ of the MSI colorectal carcinomas contain wild-type APC, they may have other defects in the Wnt pathway causing stabilization of B-catenin and thereby increased activity. For example dominant mutations in the $\beta$-catenin gene itself or the tumour suppressive Conductin/Axin-2 gene have been found which all omit efficient $B$-catenin degradation $(6,8)$.

Aberrant nuclear accumulation of $B$-catenin leads to association with transcription factors including lymphoid enhancer factor-1 (LEF-1) or T cell factors (TCF) $(11,12)$. These molecules are DNA-binding proteins driving together with B-catenin the transcription of multiple target genes such as c-myc, cyclin D1, MMP-7, laminin-5 2 implicated in proliferation, cell-cell attachment, and invasion (13-15). Collectively, aberrant Wnt-signalling and nuclear B-catenin accumulation may serve as the underlying basis for invasion, metastasis and poor prognosis $(8,15)$.

The relationship between alterations in the Wnt-signalling pathway and concomitant accumulation of nuclear B-catenin as well as differences in the $\beta$-catenin expression between CIN and MSI have not been fully clarified and divergent results have been reported (16). However, in most sporadic CRC, high degree of MSI has been reported to correlate with low frequencies of APC and B-catenin mutations and better prognosis, while CIN is associated with poor outcome and disruption of the Wnt signalling pathway $(17,18)$. 
The aim of the present study was to investigate whether nuclear accumulation of $\beta$-catenin is associated with genetic instability and prognosis. Therefore, we studied the expression of $\beta$-catenin in the clinically well characterized material of 67 CRC using immunohistochemistry and compared the results with data on DNA content, MSI/MSS status and the p53 expression status.

\section{Materials and methods}

Patient samples and tumour data. Seventy-seven patients with primary colorectal cancer were retrospectively included in this study, but 10 were excluded due to no evaluable tissue material [loss of tissue material in the antigen retrieval procedures or lack of B-catenin staining (see below) in non-tumour cells], leaving 67 patients for further analyses. The age of the patients ranged from 37 to 88 years and the male to female ratio was 3:2. Each tumour was classified according to tumour localisation within the bowel (right colon, left colon and rectum), stage, grade and growth pattern at the invasive margin (pushing or infiltrating). All tumours were operated on with a curative intent and were classified as radically removed by both the surgeon and the pathologist. Regarding ploidy, MSI and p53 expression (see below), evaluation of less than 67 tumours was possible, explaining the smaller number in these groups (Tables III and IV). This study was approved by the Ethics Committee at Umeå University, Umeå, Sweden.

Immunohistochemical procedures. CRC specimens had been fixed in formalin and embedded in paraffin wax, according to routine procedures. For this study, 4- $\mu \mathrm{m}$ sections were prepared, dried, deparaffinised and rehydrated before microwave treatment in citrate buffer ( $\mathrm{pH}$ 6.0). Antigen visualisation was performed using a semiautomatic staining machine (Ventana ES, Ventana Inc., AZ, USA). A polyclonal ß-catenin antibody (C2206; Sigma Chemical Co., St. Louis, MO, USA) was applied at a dilution of 1:750 and the slides were then counterstained with haematoxylin. Similar staining procedures were applied for p53. The antibody used was anti-p53 (Ab-6, Oncogene Research Inc., NY, USA) at a dilution of 1:400.

Evaluation of immunohistochemical staining. Slides were interpreted by one investigator, unaware of the results of the other analyses. All slides were independently reviewed twice and intraobserver disagreements $(<10 \%)$ were reviewed a third time followed by a conclusive judgment. Evaluation of the antibody was performed using a semiquantitative scale.

The entire tissue sections for each tumour were scanned to estimate the mean value of $B$-catenin positive nuclei using a three graded scale $(-),(<5 \%) ;(+),(5-50 \%)$; and $(++)$, $(>50 \%)$. At the invasive margin tumour budding $(<10$ cells) was evaluated according to the same semiquantitative scale. Concerning p53, the tumours were assigned to one of the two categories: abnormal expression, more than $25 \%$ of the cancer cells were stained; and normal expression, $0-25 \%$ of the cancer cells were stained (19).

DNA ploidy analysis. DNA-staining was performed according to Vindelof et al (20). A FACScan flow cytometer (BectonDickinson Immunocytometry Systems, Inc., San Jose, CA) was used and the DNA index (DI) was calculated. A tumour was denominated diploid (DI=1) when only one peak was detected and aneuploid when two (or more) separate peaks were found.

\section{Microsatellite instability}

DNA extraction. Tissue for extracting DNA was available from 62 out of 67 tumours. DNA was extracted from snapfrozen tumour and corresponding normal tissue collected immediately after curative surgical removal of the tissue. A minority of normal control tissue, 6 out of 67 , had to be extracted from formalin-fixed and paraffin-embedded material when snap-frozen normal tissue was missing.

From snap-frozen tissue, DNA was extracted using the Nucleon $^{\mathrm{TM}}$ ST kit (Amersham Life Science, England) according to the manufacturer's instructions. From paraffin-embedded tissue, 5-10 $\mu \mathrm{m}$ sections (depending on the size of the tissue available) were deparaffinised in xylene and then subjected to centrifugation, followed by incubation of the pellet in alcohol in order to remove any remnant of the xylene solution. The retrieved material was then incubated overnight in buffer containing $50 \mathrm{mM}$ Tris- $\mathrm{HCl}, \mathrm{pH} 8.0,1 \mathrm{mM}$ EDTA, 0.50\% Tween-20 and proteinase $\mathrm{K}(0.4 \mathrm{mg} / \mathrm{ml})$ at a temperature of $55^{\circ} \mathrm{C}$. In order to inactivate proteinase $\mathrm{K}$, the tumour material was incubated for $8 \mathrm{~min}$ at a temperature of $95^{\circ} \mathrm{C}$. The samples were analysed for microsatellite status using PCR.

PCR analysis and interpretation. Five different microsatellite markers were used to decide the MSI status of the tumours. They included two markers covering (CA) n repeats (D2S123 and D17S250) and three poly-A repeat specific markers (BAT25, BAT26 and BAT34C4). According to Boland et al (21), a tumour was scored as high-frequency MSI (MSI-H) if at least two out of five markers showed instability, while those with one out of five markers were scored as lowfrequency MSI (MSI-L) (21). Tumours with no markers showing instability were defined as microsatellite stable (MSS). The PCR reaction was performed using standard conditions with one of the primers in each pair being fluorescence-dye labelled. The PCR products were analyzed by use of an ABI 377 Auto-mated Genetic Analyzer with the help of GeneScan software.

Statistics. Differences between groups were examined using the Kruskal-Wallis test. Correlations between ordinale scale variables were examined using the linear-by-linear association test. The Kaplan-Meier method was used to estimate cancerspecific survival and comparison between groups was performed with the log-rank test. A p-value $<0.05$ was required for statistical significance and two-sided tests were performed for all analyses. Statistical analyses were done using SPSS version 11.0 (SPSS Inc., IL, USA).

\section{Results}

Immunohistochemistry. Twenty-two out of 67 tumours (33\%) lacked nuclear expression of $\beta$-catenin (-). Thirty-two tumours (48\%) expressed low levels (+) and $13(19 \%)$ tumours expressed high levels $(++)$ of nuclear $B$-catenin. At the invasive margin 36 out of 67 tumours $(54 \%)$ expressed no nuclear 
Table I. Expression of $\beta$-catenin (mean vaules) in relation to clinicopathological variables.

$\overline{\frac{\beta \text {-catenin }}{-}+\quad++}$ p-value Total (n)

\begin{tabular}{lrrrrr}
\hline Gender & & & & & \\
Male & 13 & 17 & 9 & 0.73 & 39 \\
Female & 9 & 15 & 4 & & 28
\end{tabular}

Age, years

$\begin{array}{lrrrrr}\leq 59 & 4 & 6 & - & 0.53 & 10 \\ 60-69 & 6 & 5 & 6 & & 17 \\ 70-79 & 10 & 12 & 6 & & 28 \\ >80 & 2 & 9 & 1 & & 12\end{array}$

Localization

$\begin{array}{lrrrrr}\text { Right } & 12 & 12 & 2 & 0.10 & 26 \\ \text { Left } & 5 & 9 & 7 & & 21 \\ \text { Rectum } & 5 & 11 & 4 & & 20\end{array}$

Stage

$\begin{array}{lrrrrr}\text { Dukes' A } & - & 7 & - & 0.34 & 7 \\ \text { Dukes' B } & 12 & 16 & 9 & & 37 \\ \text { Dukes' C } & 10 & 9 & 4 & & 23\end{array}$

Grade

$\begin{array}{lrrrrr}\text { Well } & - & 1 & - & 0.30 & 1 \\ \text { Moderate } & 18 & 29 & 12 & & 59 \\ \text { Poor } & 2 & 1 & & & 3\end{array}$

Tumour type

$\begin{array}{llllll}\text { Mucinous } & 10 & 6 & 1 & 0.01 & 17\end{array}$

$\begin{array}{lllll}\text { Non-mucinous } & 12 & 26 & 12 & 50\end{array}$

Growth pattern

$\begin{array}{lrrrrr}\text { Pushing } & 13 & 21 & 4 & 0.23 & 38 \\ \text { Infiltrating } & 9 & 11 & 9 & & 29\end{array}$

B-catenin (-) in areas showing tumour budding, $10(15 \%)$ expressed low (+), and $21(31 \%)$ expressed high levels (++) of $\beta$-catenin. A significant correlation was found between the mean nuclear $\beta$-catenin expression and nuclear expression in tumour budding at the invasive margin $(\mathrm{p}<0.001)$.

Clinicopathological parameters. The mean $\beta$-catenin nuclei expression in tumours did not correlate with gender, age, stage, grade, localisation or growth pattern but with tumour type $(0.01)$ as detailed in Table I. Only 1 out of 17 mucinous tumours expressed high levels of nuclear B-catenin compared with 12 out of 50 of non-mucinous tumours. Nuclear B-catenin expression in tumour budding at the invasive margin did correlate with tumour type $(\mathrm{p}=0.03)$ and growth pattern $(\mathrm{p}=0.020)$, but not with other investigated clinicopathological parameters (Table II).

Genetic instability-ploidy and MSI. The mean values of nuclear $\beta$-catenin expression showed a significant correlation
Table II. Expression of $\beta$-catenin in tumour budding at the invasive margin in relation to clinicopathological variables.

\begin{tabular}{|c|c|c|c|c|c|}
\hline & \multicolumn{3}{|c|}{ B-catenin } & \multirow[b]{2}{*}{ p-value } & \multirow[b]{2}{*}{ Total (n) } \\
\hline & - & + & ++ & & \\
\hline \multicolumn{6}{|l|}{ Gender } \\
\hline Male & 19 & 5 & 15 & 0.19 & 39 \\
\hline Female & 17 & 5 & 6 & & 28 \\
\hline \multicolumn{6}{|l|}{ Age, years } \\
\hline$\leq 59$ & 7 & - & 3 & 0.82 & 10 \\
\hline $60-69$ & 7 & 2 & 8 & & 17 \\
\hline $70-79$ & 16 & 5 & 7 & & 28 \\
\hline$>80$ & 6 & 3 & 3 & & 12 \\
\hline \multicolumn{6}{|l|}{ Localisation } \\
\hline Right & 16 & 6 & 4 & 0.21 & 26 \\
\hline Left & 9 & 3 & 9 & & 21 \\
\hline Rectum & 11 & 1 & 8 & & 20 \\
\hline \multicolumn{6}{|l|}{ Stage } \\
\hline Dukes' A & 5 & 1 & 1 & 0.44 & 7 \\
\hline Dukes' B & 19 & 6 & 12 & & 37 \\
\hline Dukes' C & 12 & 3 & 8 & & 23 \\
\hline \multicolumn{6}{|l|}{ Grade } \\
\hline Well & - & 1 & - & 0.15 & 1 \\
\hline Intermediate & 31 & 8 & 20 & & 59 \\
\hline Poor & 3 & - & - & & 3 \\
\hline \multicolumn{6}{|l|}{ Tumour type } \\
\hline Mucinous & 13 & 2 & 2 & 0.025 & 17 \\
\hline Non-mucinous & 23 & 8 & 14 & & 35 \\
\hline \multicolumn{6}{|l|}{ Growth pattern } \\
\hline Pushing & 25 & 5 & 8 & 0.020 & 25 \\
\hline Infiltrating & 11 & 5 & 13 & & 29 \\
\hline
\end{tabular}

with ploidy $(\mathrm{p}=0.02)$. All tumours expressing high amounts $(++)$ of nuclear $\beta$-catenin were aneuploid (Table III). ß-catenin expression in tumour budding at the invasive margin were also correlated with ploidy $(\mathrm{p}=0.02)$, where 17 tumours out of $18(94 \%)$ with high mean $\beta$-catenin levels were aneuploid (Tables III and IV).

MSS status was found in 56 out of 62 tumours while 6 tumours were MSI-H. No tumour was classified as MSI-L. MSI was significantly associated with low mean levels of nuclear B-catenin $(\mathrm{p}=0.02)$ and with low expression in tumour budding $(\mathrm{p}=0.03)$. Five out of $6(83 \%)$ MSI-H tumours were $ß$-catenin negative, while no such $\beta$-catenin expression pattern was found in the other group of tumours (Tables II and III).

p53. Accumulation of p53 was significantly associated with nuclear $B$-catenin expression $(\mathrm{p}=0.003)$ and with nuclear $\beta$-catenin in tumour budding $(\mathrm{p}=0.01)$. Interestingly abnormal p53 accumulation was found in 13 out of $16(81 \%)$ tumours with high mean value of $\beta$-catenin (++) and in 16 out of 21 
Table III. Expression of $\beta$-catenin (mean values) in relation to ploidy, microsatellite instability status and p53 status.

\begin{tabular}{lrrrrr}
\hline & \multicolumn{3}{c}{ B-catenin } & & \\
\cline { 2 - 3 } & - & + & ++ & p-value & Total (n) \\
\hline Ploidy & & & & & \\
$\quad$ Aneuploid & 10 & 17 & 11 & 0.02 & 38 \\
$\quad$ Diploid & 8 & 8 & - & & 16 \\
MSI & & & & & \\
$\quad$ None & 16 & 27 & 13 & 0.02 & 56 \\
$\quad$ High & 5 & 1 & - & & 6 \\
p53 & & & & & \\
$\quad \leq 25 \%$ & 16 & 11 & 3 & 0.003 & 30 \\
$>25 \%$ & 6 & 20 & 10 & & 36 \\
\hline
\end{tabular}

Table IV. Expression of B-catenin in tumour budding at the invasive margin in relation to ploidy, microsatellite instability status and p53 status.

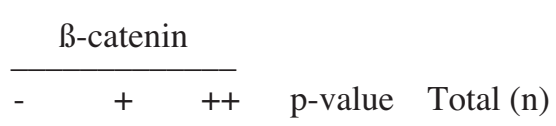

\begin{tabular}{lrrrrr}
\hline Ploidy & & & & & \\
$\quad$ Aneuploid & 17 & 4 & 17 & 0.02 & 38 \\
$\quad$ Diploid & 11 & 4 & 1 & & 16 \\
MSS/MSI & & & & & \\
MSS & 28 & 7 & 21 & 0.03 & \\
MSI-L & - & - & - & & 56 \\
MSI-H & 6 & - & - & & 6 \\
p53 & & & & & \\
Normal & 22 & 3 & 5 & 0.004 & 30 \\
Accumulation & 13 & 7 & 16 & & 36 \\
\hline
\end{tabular}

(76\%) tumours with high expression in tumour budding at the invasive margin (Tables II and IV).

Survival analyses. Mean nuclear B-catenin expression levels did not correlate with patient survival when comparing all Dukes' stages $(\mathrm{p}=0.62)$ with all three expression levels of $\beta$-catenin (Fig. 1), nor when sub-grouping the $\beta$-catenin expression into the two sub-groups high vs. negative as well as low level $(\mathrm{p}=0.44)$. However, when separately analysing the clinically most important prognostic group, Dukes' stage B patients, nuclear $B$-catenin expression (high vs. low and negative) turned out to be significantly related to patient prognosis $(p=0.04)$. Tumours with high nuclear $\beta$-catenin expression had a poorer prognosis vs. tumours with low or negative expression for $\mathrm{B}$-catenin combined ( $\mathrm{p}=0.01$; Fig. 2 ).

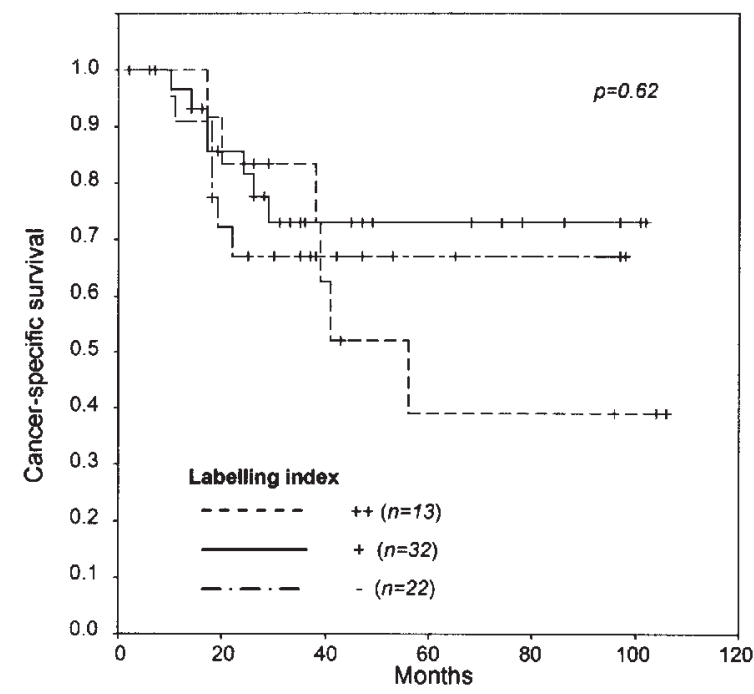

Figure 1. Kaplan-Meier cancer-specific survival curves for $67 \mathrm{CRC}$ patients, grouped according to their mean values of nuclear $\beta$-catenin staining patterns ,-+ and ++ .

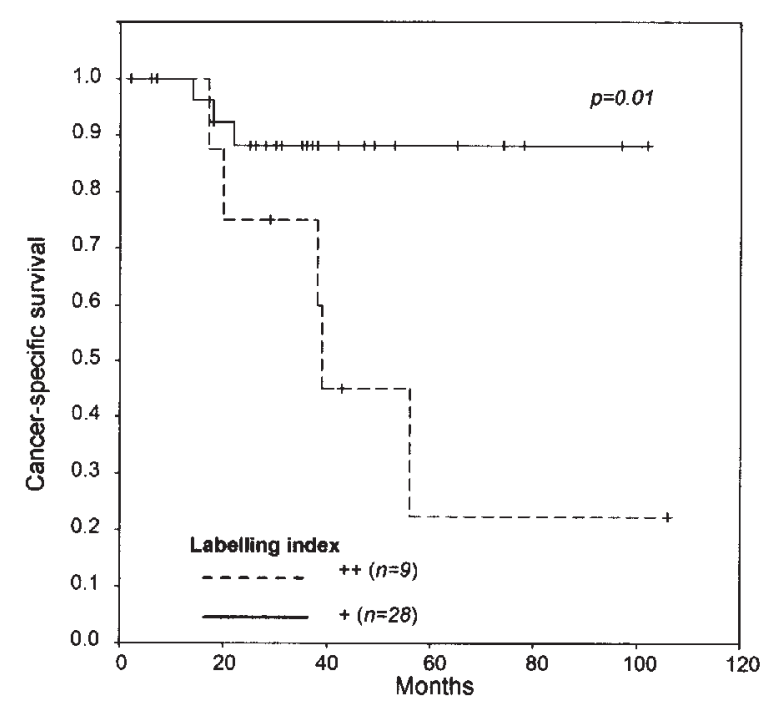

Figure 2. Kaplan-Meier cancer-specific survival curves for 37 CRC patients with Dukes' B tumours, grouped according to their mean values of nuclear ß-catenin staining patterns -/+ vs. ++.

Although not statistically significant, the overall prognostic trend was similar studying $\beta$-catenin expression in tumour budding at the invasive margin. Including all Dukes' stages the three expression levels of $B$-catenin were not significantly associated with prognosis $(\mathrm{p}=0.28)$, neither when analyzing high $\beta$-catenin expression vs. negative and low levels combined $(\mathrm{p}=0.11)$. In the group of Dukes' B patients a $\mathrm{p}$-value of 0.092 was recorded (high vs. low and negative groups combined).

\section{Discussion}

Dysfunction in the Wnt-signalling pathway, and thereby nuclear accumulation of $\beta$-catenin, is thought to be an important step in the carcinogenesis of colorectal cancer and other malignant tumours (6). B-catenin expression due to APCmutation and development of CIN are accepted elements in 
the tumourigenesis of $\mathrm{CRC}$, but the part nuclear B-catenin expression plays is still not clear (22).

In the present study we used immunohistochemical staining to study nuclear $\beta$-catenin expression in tumours already characterized with respect to microsatellite and chromosomal instability as well as p53 accumulation. In order to optimise technical conditions and reproducibility, we used a semiautomatic immunohistochemical staining machine for all staining procedures.

Our results show significant association between high expression of nuclear $\beta$-catenin, aneuploidy and abnormal accumulation of $\mathrm{p} 53$, which is in line with previous reports $(2,16)$. Furthermore, in the clinically most interesting prognostic group, Dukes' B tumours, we found that high nuclear B-catenin expression is significantly associated with a poor prognosis. Similar findings have been reported previously (23-25), whereas Roca et al have reported contradictory observations (26).

There is also evidence that the amount of tumour budding at the invasive margin is associated with increased risk of death in CRC $(27,28)$ and budding probably mirrors tumour behaviour associated with invasiveness $(29,30)$. B-catenin has been shown to be expressed mostly at the invasive margin in most CRCs and seems to correlate with the amount of budding $(17,31)$. In analogy, high nuclear accumulation of ß-catenin in these cell buds might give the tumour cells the ability to detach, migrate and disseminate (8). In the present patient material we have also evaluated the value of $\beta$-catenin expression levels at the invasive margin. The prognostic results are in agreement with mean tumour expression level of $\beta$-catenin, although not statistically significant. Thus the expression levels of $B$-catenin may be an insufficient marker for the biological behaviour of colorectal cancers. It has been shown that inactive or phosphorylated B-catenin correlates with a better prognosis (32). Consequently the activation status of $\beta$-catenin has the potential of adding more prognostic information and should be further studied.

In addition, tumours with p53 mutations as well as aneuploid tumours are known to be associated with a slightly poorer prognosis (33). In contrast, MSI tumours were significantly related with lack of nuclear ß-catenin expression and normal p53 expression (16). Lack of nuclear B-catenin expression tended $(\mathrm{p}=0.10)$ to be correlated to right sided tumour localisation, which is in agreement with several studies and known clinicopathological features. Another known clinical feature is that MSI CRCs tend to be late stage at presentation, but to have a better prognosis than CIN CRCs (34). The results in our survival analyses is in accordance with such a view, though the number of MSI cases in the present study is too small to establish conclusive results. However, the important question remains, if the lack of nuclear B-catenin expression renders MSI tumours less aggressive or whether other alterations in the tumourigenesis inhibit promotion of late stage lesions. Absence of nuclear B-catenin expression in MSI CRCs is consistent with previous studies (6). These tumours have been shown to have more often other mutations in the Wnt-signalling pathway than in the APC-gene, for example in exon 3 of B-catenin gene or Axin $(35,36)$.
In conclusion, we have shown that CIN CRCs are frequently associated with high nuclear $\beta$-catenin expression, DNA-aneuploidy and abnormal p53 expression, whereas low/negative nuclear $\beta$-catenin expression, normal p53 expression seem to occur in MSI CRCs. In the survival analyses a significant poor prognosis was recorded for Dukes' $B$ tumours with high nuclear expression of $\beta$-catenin. The clinical use of $\beta$-catenin as a prognostic factor needs to be investigated in larger patient cohorts.

\section{Acknowledgements}

The study was supported by grants from the Swedish Cancer Society (2520-B04-18XAB) and the Lion's Cancer Research Foundation in Umeå. The authors thank Mrs. Kerstin Näslund for skilful technical assistance.

\section{References}

1. Tomlinson I, Ilyas M and Novelli M: Molecular genetics of colon cancer. Cancer Metastasis Rev 16: 67-79, 1997.

2. Fernebro E, Halvarsson B, Baldetorp B, et al: Predominance of CIN versus MSI in the development of rectal cancer at young age. BMC Cancer 2: 25, 2002.

3. Tomlinson I and Bodmer W: Selection, the mutation rate and cancer: ensuring that the tail does not wag the dog. Nat Med 5: 11-12, 1999.

4. Rajagopalan H, Nowak MA, Vogelstein B, et al: The significance of unstable chromosomes in colorectal cancer. Nat Rev Cancer 3: 695-701, 2003.

5. Lengauer C, Kinzler KW and Vogelstein B: Genetic instabilities in human cancers. Nature 396: 643-649, 1998.

6. Shimizu Y, Ikeda S, Fujimori M, et al: Frequent alterations in the Wnt signaling pathway in colorectal cancer with microsatellite instability. Genes Chromosomes Cancer 33: 73-81, 2002.

7. Lengauer C, Kinzler KW and Vogelstein B: Genetic instability in colorectal cancers. Nature 386: 623-627, 1997.

8. Brabletz T, Jung A and Kirchner T: Beta-catenin and the morphogenesis of colorectal cancer. Virchows Arch 441: 1-11, 2002.

9. Morin PJ: beta-catenin signaling and cancer. Bioessays 21: 1021-1030, 1999.

10. Jass JR, Young J and Leggett BA: Evolution of colorectal cancer: change of pace and change of direction. J Gastroenterol Hepatol 17: 17-26, 2002.

11. Henderson BR, Galea M, Schuechner S, et al: Lymphoid enhancer factor-1 blocks adenomatous polyposis coli-mediated nuclear export and degradation of beta-catenin. Regulation by histone deacetylase 1. J Biol Chem 277: 24258-24264, 2002.

12. Bienz M: The subcellular destinations of APC proteins. Nat Rev Mol Cell Biol 3: 328-338, 2002.

13. Takahashi M, Tsunoda T, Seiki M, et al: Identification of membrane-type matrix metalloproteinase- 1 as a target of the beta-catenin/Tcf4 complex in human colorectal cancers. Oncogene 21: 5861-5867, 2002.

14. Cullingworth J, Hooper ML, Harrison DJ, et al: Carcinogeninduced pancreatic lesions in the mouse: effect of Smad4 and Apc genotypes. Oncogene 21: 4696-4701, 2002.

15. Wang HL, Wang J, Xiao SY, et al: Elevated protein expression of cyclin D1 and Fra-1 but decreased expression of c-Myc in human colorectal adenocarcinomas overexpressing beta-catenin. Int J Cancer 101: 301-310, 2002.

16. Soussi T: The p53 tumour suppressor gene: from molecular biology to clinical investigation. Ann NY Acad Sci 910: 121-137, 2000 .

17. Jass JR, Barker M, Fraser L, et al: APC mutation and tumour budding in colorectal cancer. J Clin Pathol 56: 69-73, 2003.

18. Gafa R, Maestri I, Matteuzzi M, et al: Sporadic colorectal adenocarcinomas with high-frequency microsatellite instability. Cancer 89: 2025-2037, 2000.

19. Baas IO, Mulder JW, Offerhaus GJ, et al: An evaluation of six antibodies for immunohistochemistry of mutant p53 gene product in archival colorectal neoplasms. J Pathol 172: 5-12, 1994.

20. Vindelof LL, Christensen IJ, Nissen NI, et al: A detergent-trypsin method for the preparation of nuclei for flow cytometric DNA analysis. Cytometry 3: 323-327, 1983. 
21. Boland CR, Thibodeau SN, Hamilton SR, et al: A National Cancer Institute Workshop on Microsatellite Instability for cancer detection and familial predisposition: development of international criteria for the determination of microsatellite instability in colorectal cancer. Cancer Res 58: 5248-5257, 1998.

22. Lamlum H, Papadopoulou A, Ilyas M, et al: APC mutations are sufficient for the growth of early colorectal adenomas. Proc Natl Acad Sci USA 97: 2225-2228, 2000.

23. Bondi J, Bukholm G, Nesland JM, et al: Expression of nonmembranous beta-catenin and gamma-catenin, c-Myc and cyclin D1 in relation to patient outcome in human colon adenocarcinomas. APMIS 112: 49-56, 2004.

24. Cheah PY, Choo PH, Yao J, et al: A survival-stratification model of human colorectal carcinomas with beta-catenin and p27kip1. Cancer 95: 2479-2486, 2002.

25. Baldus ES, Mönig PS, Huxel S, et al: MUC1 and nuclear B-catenin are coexpressed at the invasion front of colorectal carcinomas and are both correlated with tumor prognosis. Clin Cancer Res 10: 2790-2796, 2004.

26. Roca F, Mauro VL, Bonadeo F, et al: Prognostic value of E-cadherin, beta-catenin, MMPs (7 and 9) and TIMPs (1 and 2) in patients with colorectal carcinoma. J Surg Oncol 93: 151-160, 2006.

27. Cianchi F, Messerini L, Palomba A, et al: Character of the invasive margin in colorectal cancer: does it improve prognostic information of Dukes staging? Dis Colon Rectum 40: 1170-1175, 1997.

28. Park KJ, Choi HJ, Roh MS, et al: Intensity of tumor budding and its prognostic implications in invasive colon carcinoma. Dis Colon Rectum 48: 1597-1602, 2005.
29. Palmqvist R, Rutegard JN, Bozoky B, et al: Human colorectal cancers with an intact $\mathrm{p} 16 /$ cyclin $\mathrm{D} 1 / \mathrm{pRb}$ pathway have upregulated p16 expression and decreased proliferation in small invasive tumour clusters. Am J Pathol 157: 1947-1953, 2000.

30. Ueno H, Murphy J, Jass JR, et al: Tumour 'budding' as an index to estimate the potential of aggressiveness in rectal cancer. Histopathology 40: 127-132, 2002.

31. Brabletz T, Jung A, Hermann K, et al: Nuclear overexpression of the oncoprotein beta-catenin in colorectal cancer is localized predominantly at the invasion front. Pathol Res Pract 194: 701-704, 1998.

32. Chung GG, Provost E, Kielhorn EP, et al: Tissue microarray analysis of $ß$-catenin in colorectal cancer shows nuclear phosphoB-catenin is associated with a better prognosis. Clin Cancer Res 7: 4013-4020, 2001

33. Kapiteijn E, Liefers GJ, Los LC, et al: Mechanisms of oncogenesis in colon versus rectal cancer. J Pathol 195: 171-178, 2001.

34. Tomlinson I: Different pathways of colorectal carcinogenesis and their clinical pictures. Ann NY Acad Sci 910: 10-18, 2000.

35. Sparks AB, Morin PJ, Vogelstein B, et al: Mutational analysis of the APC/beta-catenin/Tcf pathway in colorectal cancer. Cancer Res 58: 1130-1134, 1998.

36. Miyaki M, Iijima T, Kimura J, et al: Frequent mutation of betacatenin and APC genes in primary colorectal tumours from patients with hereditary nonpolyposis colorectal cancer. Cancer Res 59: 4506-4509, 1999. 